\title{
AVALIAÇÃO DA HOMOGENEIZAÇÃO DE LIGA Ti35Nb PROCESSADA POR METALURGIA DO PÓ E EXTRUSÃO ANGULAR EM CANAL*
}

\author{
Juan Garcia de Blas ${ }^{1}$ \\ Juliana do Nascimento Lunz $^{2}$ \\ Laercio Rosignoli Guzela ${ }^{3}$ \\ Alexandre Antunes Ribeiro ${ }^{4}$ \\ Luiz Carlos Pereira ${ }^{6}$ \\ Marize Varella de Oliveira ${ }^{6}$ \\ Cássio Barbosa ${ }^{7}$
}

\begin{abstract}
Resumo
O presente trabalho aborda o desenvolvimento de liga titânio-nióbio obtida por metalurgia do pó no qual foi introduzida uma operação de extrusão angular em canal (Equal Channel Angular Pressing - ECAP) com o propósito de promover uma consolidação que favorecesse a homogeneização na etapa posterior de sinterização. Após o processamento o material foi caracterizado por microscopia ótica e microscopia eletrônica de varredura (MEV/EDS). Os resultados preliminares indicaram a importância da redução do tamanho das partículas de nióbio.

Palavras-chave: Liga Titânio-Nióbio; Metalurgia do Pó; Extrusão Angular em Canal.

\section{HOMOGENIZATION ASSESSMENT ON Ti35Nb ALLOY PROCESSED BY POWDER METALLURGY AND EQUAL CHANNEL ANGULAR PRESSING}

\begin{abstract}
The present work describes the development of titanium-niobium alloy obtained by powder metallurgy introducing an equal channel angular pressing operation with the purpose of promoting consolidation that can favor homogenization in the following sinterization stage. After processing the material was characterized by optical microscopy and scanning electron microscopy (SEM/EDS). Preliminary results indicated the importance of the reduction of the niobium particles size.

Keywords: Titanium-Niobium Alloy; Powder Metallurgy; Equal Channel Angular Pressing.
\end{abstract}

1 Engenheiro Metalúrgico, Dr.Ing., Professor Associado, PEMM/COPPE, Universidade Federal do Rio de Janeiro (UFRJ), Rio de Janeiro, RJ, Brasil.

2 Química, D.Sc., Bolsista, DPCM/LATEP, Instituto Nacional de Tecnologia - INT, Rio de Janeiro, $R J$, Brasil

3 Matemático, M.Sc.Técnico de nível superior, PEMM/COPPE, Universidade Federal do Rio de Janeiro (UFRJ), Rio de Janeiro, RJ, Brasil.

4 Engenheiro Químico, D.Sc., Tecnologista, DPCM/LATEP, Instituto Nacional de Tecnologia - INT, Rio de Janeiro, RJ, Brasil.

5 Engenheiro Metalúrgico, D.Sc., Professor Associado, PEMM/COPPE, Universidade Federal do Rio de Janeiro (UFRJ), Rio de Janeiro, RJ, Brasil.

6 Engenheira Metalúrgica, D.Sc., Tecnologista, DPCM/LATEP, Instituto Nacional de Tecnologia INT, Rio de Janeiro, RJ, Brasil.

7 Engenheiro Metalúrgico, D.Sc.,Tecnologista, DEMP/LACPM, Instituto Nacional de Tecnologia INT, Rio de Janeiro, RJ, Brasil. 


\section{INTRODUÇÃO}

Recentemente tem aumentado o uso de titânio e suas ligas para a fabricação de implantes cirúrgicos ortopédicos e dentais, pois apresentam excelente biocompatibilidade, proporcionando melhor osteointegração (integração ao tecido ósseo) e menor risco de alergia e reações adversas com o organismo do que outros materiais metálicos, como, por exemplo, o aço inoxidável austenítico AISI-SAE $316 \mathrm{~L}$, cujo elevado teor de níquel pode provocar reações alérgicas. Outra vantagem do titânio e de suas ligas é o menor módulo de elasticidade (rigidez), mais próximo do osso humano do que o do aço inoxidável. A resistencia ao desgaste é uma propriedade a ser considerada na concepção das ligas de titânio no sentido de evitar a geração de fragmentos que provocam reações adversas no organismo humano, a exemplo da metalose. [1-11].

A utilização de processos de metalurgia do pó na fabricação de produtos em ligas de titânio justifica-se pelas suas vantagens econômicas. Entre elas, podem ser citadas citadas [12]: o alto aproveitamento da matéria prima com vantagem econômica, baixa energia de transformação, devido a baixas temperaturas em relação aos processos de fusão, ótimas tolerâncias dimensionais, possibilidade de ótimo acabamento superficial e permite as mais variadas combinações de elementos químicos (ligas), assim como o controle da composição química.

Por outro lado, a aplicação da deformação plástica severa atraves da extrusão angular em canal (Equal Channel Angular Pressing - ECAP) como uma etapa do processamento de pós metálicos vem sendo estudada. Verificou-se que quando as partículas são cisalhadas sua superfície é aumentada. Desta maneira, ocorre o rompimento da camada de óxido superficial e a adesão entre as particulas através das superfícies recem geradas. Esta maior continuidade nas interfaces entre particulas, além de reduzir a porosidade, favorecerá a difusão na etapa posterior de sinterização. $[13,14]$

O objetivo deste trabalho é desenvolver e caracterizar uma liga Ti-35Nb fabricada por uma conjugação de metalurgia do pó e extrusão angular em canal, visando a obtenção de um produto com biocompatibilidade e propriedades mecânicas adequadas, módulo de elasticidade inferior ao de outras ligas de titânio e assim ainda mais próximo do osso humano e resistência ao desgaste superior aos produtos fabricados por outros processos usualmente utilizados para a produção de implantes cirúrgicos. Para a caracterização do material foram utilizadas as técnicas de microscopia ótica e microscopia eletrônica de varredura (MEV) com detector de energia dispersiva de raios $X$ (EDS).

\section{MATERIAIS E MÉTODOS}

O material estudado consistiu em uma mistura de pós elementares. Após peneiramento, o pó de Ti (grau 1 com 99,5\% de pureza) apresentava partículas com tamanho entre 30 e $150 \mu \mathrm{m}$ e o pó de $\mathrm{Nb}(99.8 \%$ de pureza), partículas com tamanho acima de $150 \mu \mathrm{m}$. A mistura dos pós, na proporção Ti-35\% Nb, foi realizada em misturador Retsch MM400 com duração de 15 min e frequência de $15 \mathrm{~s}^{-1}$. Após a mistura e homogeneização os pós foram submetidos à compactação uniaxial à temperatura ambiente, com pressão de $730 \mathrm{MPa}$, obtendo-se pastilhas cilíndricas com diâmetro de $6,3 \mathrm{~mm}$, segundo procedimento empregado em trabalhos anteriores [15-16]. 
Os corpos de prova para obtenção de material processado por ECAP consistiram num invólucro de aço 1020 preenchido com três pastilhas compactadas perfazendo um comprimento total de $25,4 \mathrm{~mm}$, mostrado esquematicamente na Fig. 1. Para a aplicação do ECAP foi utilizada uma matriz com canais com seção quadrada de $13,1 \times 13,1 \mathrm{~mm}$ interceptando-se num ângulo $\Phi$ de $90^{\circ}$ e com transição na aresta externa compreendida num ângulo $\Psi$ de $20^{\circ}$. Nestas condições, a deformação verdadeira por passe é de 1,05 [17]. Os ensaios foram realizados a $375^{\circ} \mathrm{C}$ sob uma taxa de deformação de aproximadamente $0,5 \mathrm{~s}^{-1}$ e consistiram em dois passes conduzidos segundo a rota C [18], na qual é aplicada uma rotação de $180^{\circ}$ ao corpo de prova entre os passes, o que provoca a inversão do sentido da deformação.

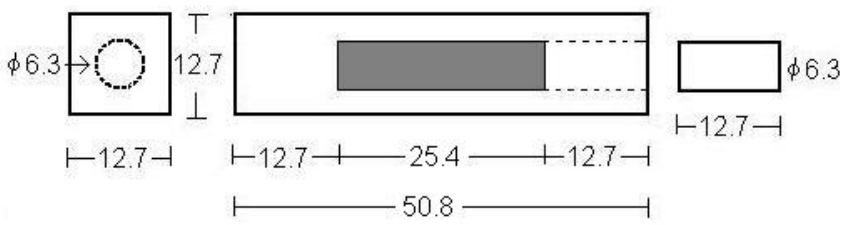

Figura 1. Representação esquemática do corpo de prova para ECAP. Dimensões em mm.

Após processamento por ECAP, as pastilhas, formando um bloco consolidado, foram retiradas do invólucro de aço e cortadas na seção transversal com disco adiamantado obtendo-se amostras que posteriormente foram submetidas a tratamentos de sinterização em forno Thermal Technology LLC sob vácuo de $10^{-6}$ Torr em diferentes temperaturas: $900,1000,1100,1200$ e $1300^{\circ} \mathrm{C}$, com taxa de aquecimento de $10^{\circ} \mathrm{C} / \mathrm{min}$, duração de $2 \mathrm{~h}$ e posterior resfriamento em forno, a fim de promover a homogeneização do material.

A caracterização microestrutural dos materiais processados foi realizada por microscopia ótica e MEV-EDS. A observação por MEV-EDS das amostras sinterizadas foi realizada sobre a superfície resultante do tratamento. A preparação das amostras submetidas às demais condições consistiu em corte, embutimento, lixamento e polimento convencionais. A densidade das amostras compactadas foi determinada pelo método geométrico [19].

\section{RESULTADOS E DISCUSSÃO}

O aspecto típico de uma amostra compactada à temperatura ambiente é mostrado pela imagem de MEV da Figura 2. Pode-se observar que as partículas de $\mathrm{Nb}$, distinguíveis pela sua coloração clara e seu mapa de EDS, apresentam uma distribuição uniforme e seu tamanho médio é superior ao das partículas de Ti. Notase ainda a existência de porosidade entre partículas, aspecto compatível com o valor medido para a densidade relativa de $86 \%$.

$\mathrm{Na}$ Figura 3 são mostradas imagens de microscopia ótica obtidas em amostras processadas por ECAP. Após o primeiro passe (Fig. 3a) as partículas de ambos os metais apresentam-se alongadas. Como, devido ao emprego da rota $\mathrm{C}$, a deformação do segundo passe foi realizada no sentido contrário, as partículas retomam aproximadamente sua forma original (Fig. 3b). Este comportamento dútil favorece a formação de uma interface contínua entre as partículas, notadamente entre aquelas com diferente composição, fato que pode facilitar a homogeneização numa subsequente etapa de sinterização. O aumento da densidade relativa de pós consolidados por ECAP, cujo valor pode superar 99\% [15], também atuará neste sentido. 

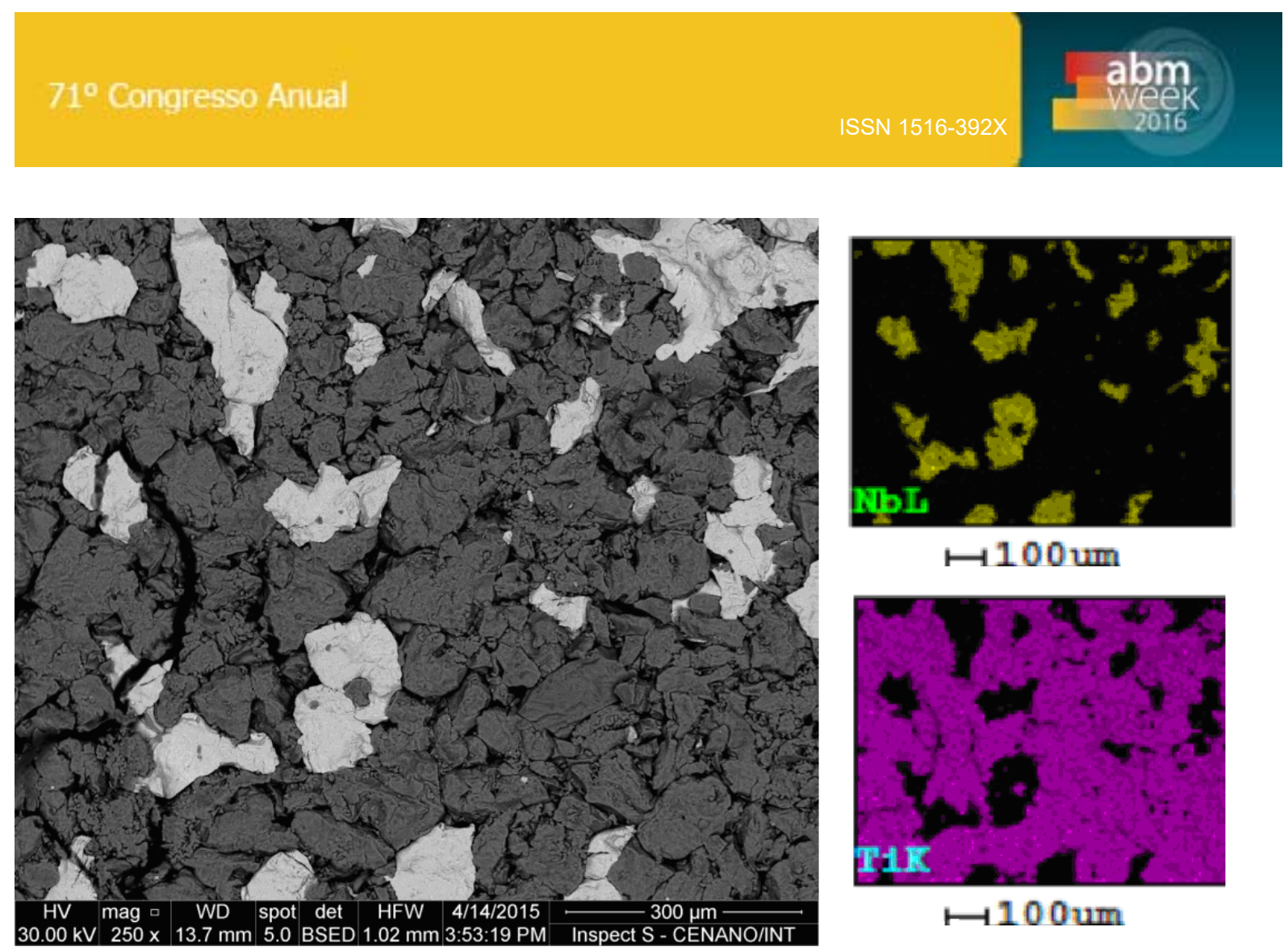

$\mapsto 100$ um

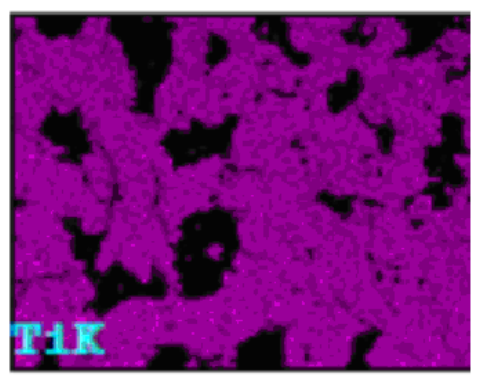

$\mapsto 100 u m$

Figura 2. Imagem da amostra compactada de liga Ti-35Nb em MEV e mapas de EDS: a matriz escura é titânio e as partículas claras são nióbio.
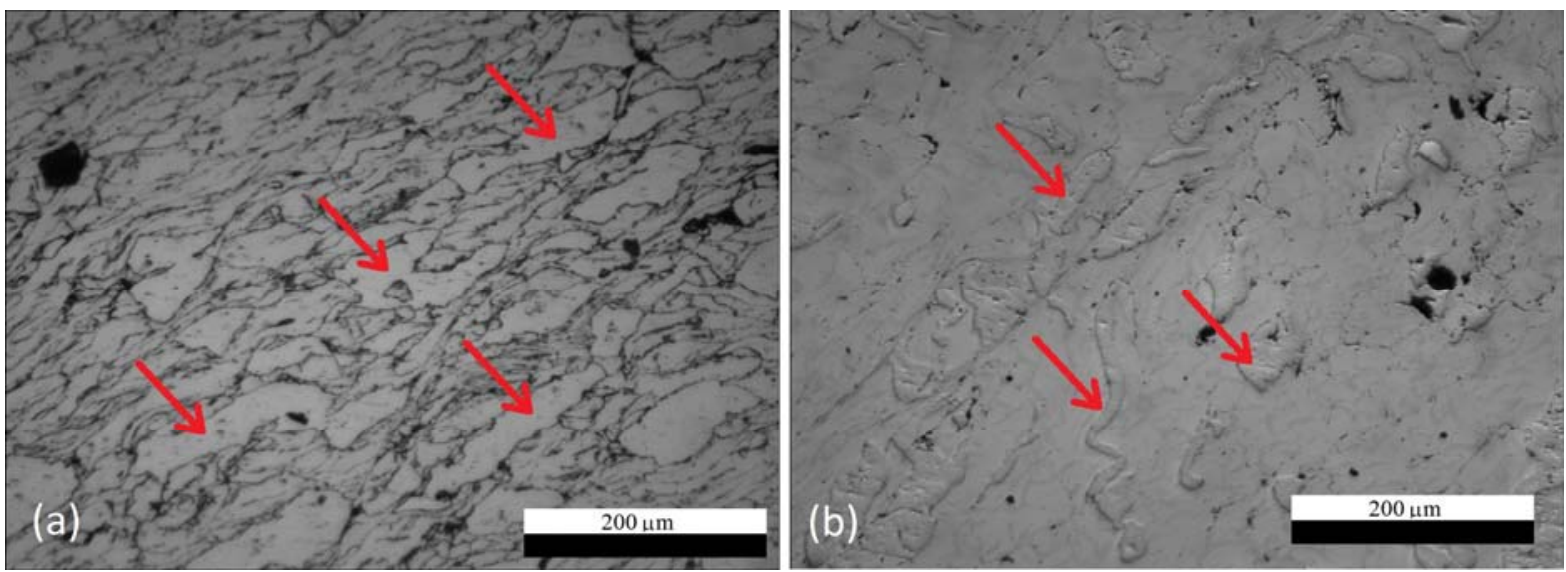

Figura 3. Aspecto das amostras processadas por ECAP após 1 passe (a) e 2 passes (b). MO sem ataque, contraste de interferência. São indicadas com setas algumas partículas de $\mathrm{Nb}$.

O tratamento de sinterização, como esperado, apresentou resultados variados em função da temperatura. A Figura 4 apresenta uma imagem MEV da superfície sem polimento da amostra sinterizada a $900^{\circ} \mathrm{C}$. Pode-se observar a existência de duas regiões que se distinguem pelo aspecto superficial e pela composição química. A região predominante, com superfície facetada, corresponde à área ocupada inicialmente pelas partículas de titânio, como revela o espectro EDS obtido na posição 1. As zonas restantes, com aparência rugosa e ilhadas, resultam da dissolução das partículas de nióbio, fato confirmado pelo espectro EDS obtido na posição 2. No que se segue, a região predominante será referida como "matriz" e as áreas ricas em nióbio serão referidas como "zonas". 

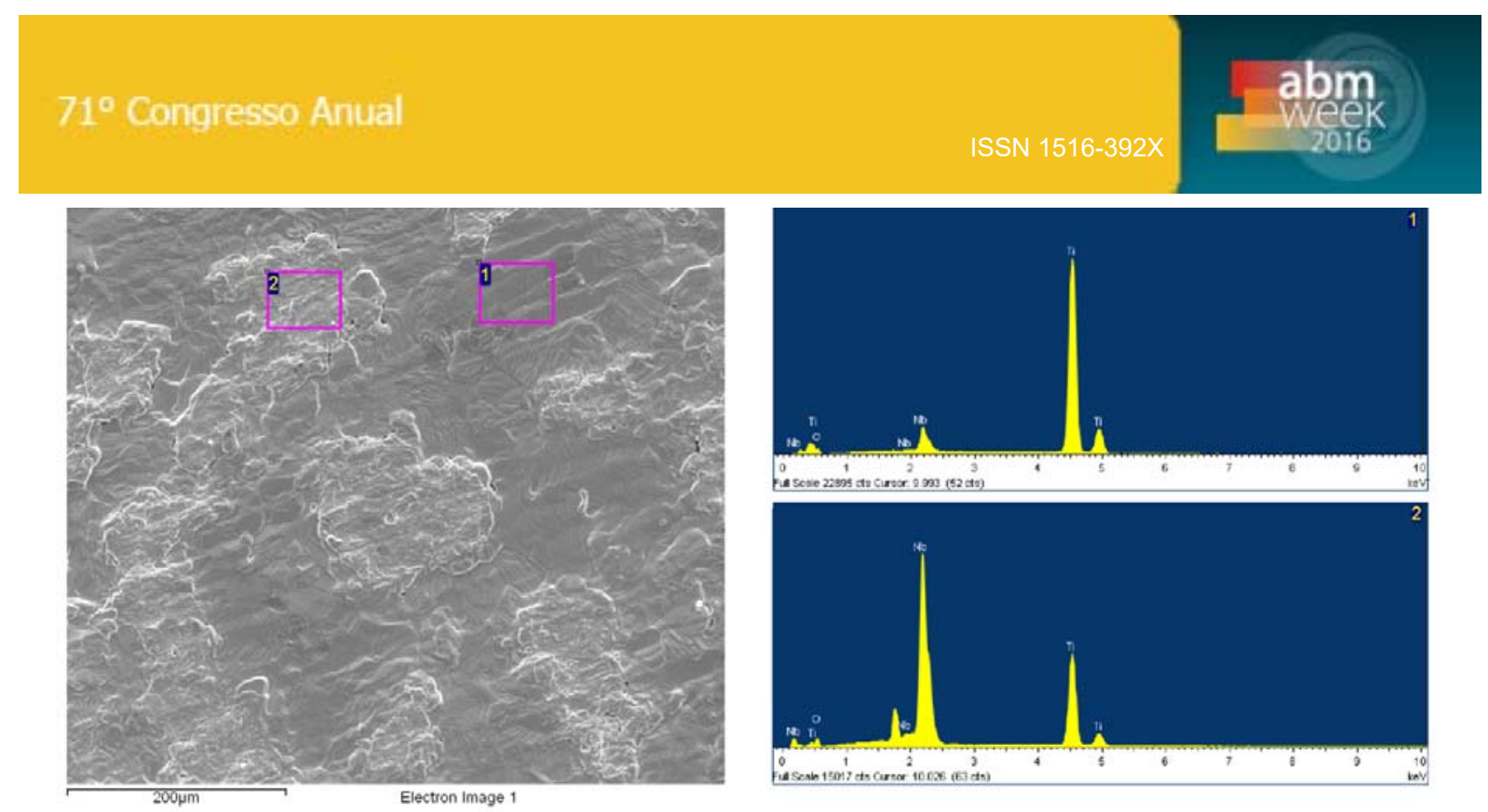

Figura 4. Imagem em MEV da amostra submetida a 2 passes de ECAP e sinterizada a $900^{\circ} \mathrm{C}$ e espectros EDS correspondentes às posições indicadas.

A microestrutura produzida pela sinterização a $1200^{\circ} \mathrm{C}$ pode ser observada na Figura 5. Observa-se a existência de uma única fase, com aspecto típico da fase $\beta$ [20], sendo que nas áreas da matriz percebem-se grãos bem definidos (intercepto médio $67 \pm 13 \mu \mathrm{m}$ ) e as zonas ricas em $\mathrm{Nb}$ distinguem-se pelo seu relevo. Os espectros de EDS mostram o progresso da homogeneização, com relação à sinterização a $900^{\circ} \mathrm{C}$ (Fig. 4): o aumento do teor de $\mathrm{Nb}$ na matriz e o de $\mathrm{Ti}$ nas zonas.
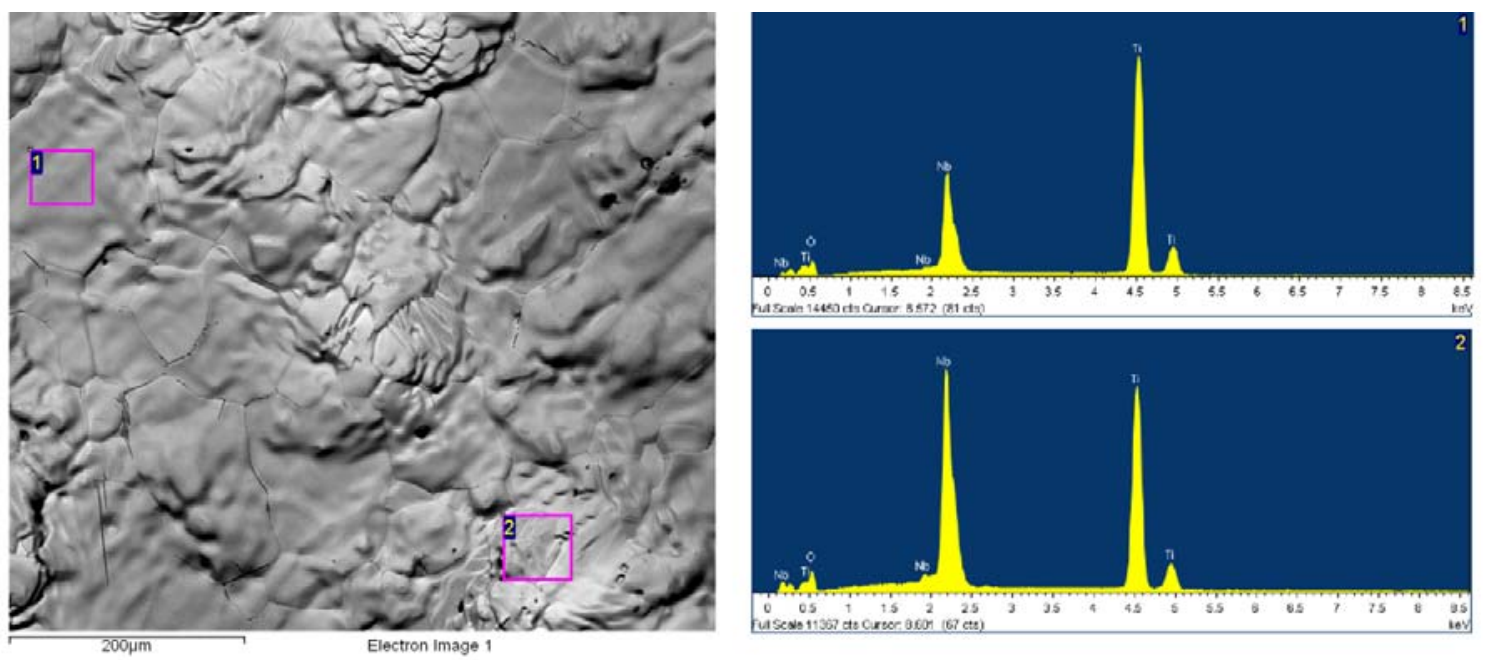

Figura 5. Imagem em MEV da amostra submetida a 2 passes de ECAP e sinterizada a $1200^{\circ} \mathrm{C}$ e espectros EDS correspondentes às posições indicadas.

Finalmente, a amostra sinterizada a $1300^{\circ} \mathrm{C}$ apresentou uma microestrutura homogênea cuja composição química medida por EDS coincide com o valor esperado para a liga, além da ocorrência de um acentuado crescimento dos grãos, com intercepto médio $94 \pm 12 \mu \mathrm{m}$.

Um aspecto observado nas amostras sinterizadas foi a ausencia de poros. A porosidade do material compactado a frio foi eliminada pela deformação imposta no processamento por ECAP. A título de comparação, um material preparado em condições similares e sinterizado a $1200^{\circ} \mathrm{C}$ após compactação a frio apresentou uma densidade relativa de $83 \%$ [21]. 
A evolução do processo de homogeneização nas amostras sinterizadas nas diferentes temperaturas entre 900 e $1200^{\circ} \mathrm{C}$ pode ser avaliada com base nos resultados semiquantitativos dos teores de titânio e nióbio, obtidos pelo EDS. A Figura 6 mostra os valores do teor de $\mathrm{Nb}$, em função da temperatura, para as regiões da matriz e das zonas. Pode-se observar que, de maneira geral, com o aumento da temperatura de sinterização, o teor de $\mathrm{Nb}$ aumenta na matriz e diminui nas zonas, tendendo ao valor esperado para a liga, 35\%. Note-se que, já nas temperaturas mais baixas, o teor de $\mathrm{Nb}$ nas zonas é inferior ao das partículas no inicio do processo (100\%). Isto indica que, além da difusão do $\mathrm{Nb}$ em direção à matriz, também ocorreu difusão do Ti em direção às zonas.

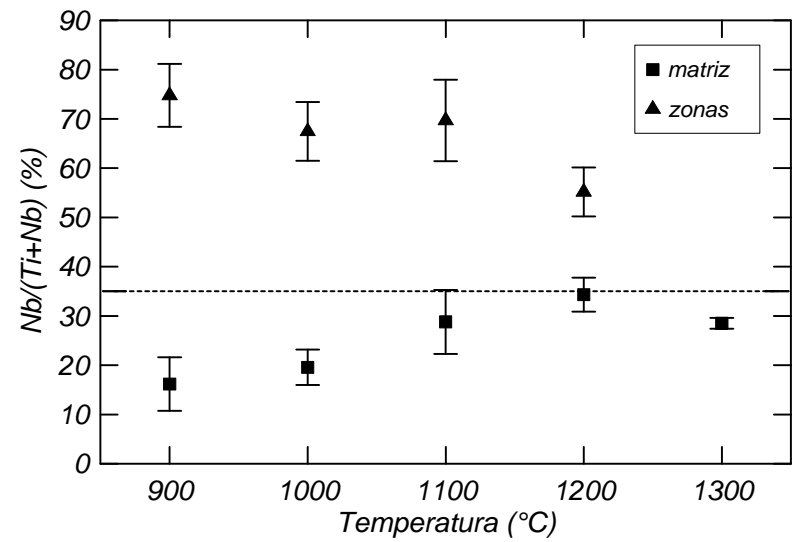

Figura 6. Teor de $\mathrm{Nb}$ medido por EDS nas diferentes regiões das amostras sinterizadas em função da temperatura de sinterização.

Embora na temperatura de $1200^{\circ} \mathrm{C}$ o teor de $\mathrm{Nb}$ nas zonas ainda seja superior a $35 \%$, pode-se considerar que a liga está próxima da homogeneização completa. Uma estimativa do grau de homogeneização do sistema pode ser feita considerando-se a matriz e as zonas como sendo "fases" de uma liga com composição global $65 \% \mathrm{Ti}-35 \% \mathrm{Nb}$ e aplicando-se a "regra da alavanca". A Figura 7 mostra os valores calculados para a fração em massa da "fase matriz", rica em Ti. A linha azul indica a fração inicial desta "fase" $(0,65)$ e a homogeneização total ocorre para o valor 1,0 . Pode-se observar que a $1200^{\circ} \mathrm{C}$ o material é praticamente composto por uma matriz homogênea. As zonas observadas nesta temperatura são remanescentes das maiores partículas de $\mathrm{Nb}$.

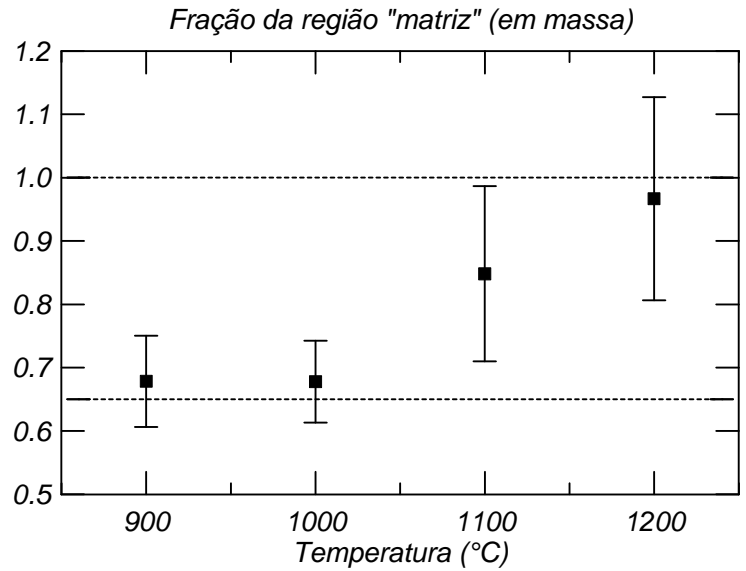

Figura 7. Estimativa da fração em massa da região matriz em função da temperatura de sinterização. 
Estes resultados mostram que a conclusão do processo de homogeneização é determinada pelas maiores partículas de $\mathrm{Nb}$. Com efeito, quanto maiores forem estas partículas, maior será a distancia média entre elas e consequentemente maiores tempos serão necessários para que o processo de difusão uniformize a concentração. Tendo em vista a importância do efeito do tamanho de grão sobre as propriedades mecânicas, é interessante a utilização de temperaturas de sinterização mais baixas, desde que a homogeneização da liga seja adequada. Na continuidade desta pesquisa será estudado o efeito da redução do tamanho das partículas de $\mathrm{Nb}$.

\section{CONCLUSÃO}

Os resultados obtidos neste estudo permitiram deduzir as seguintes conclusões:

- O processo ECAP apresentou alta eficiência na redução da porosidade e proporcionou considerável aumento de continuidade de contato entre as partículas, favorecendo significativamente a consolidação da liga obtida pela mistura e compactação de pós de titânio e de nióbio.

- Deste modo, o processo ECAP, posteriormente seguido por uma etapa de sinterização, que também contribuiu para melhorar a homogeneidade de composição química da liga Ti-Nb, foi bastante eficaz na consolidação da liga, o que pode contribuir para o aumento da resistência mecânica e resistência ao desgaste do material.

- O processamento por ECAP seguido de sinterização a $1200^{\circ} \mathrm{C}$ produziu um material composto por matriz mais homogênea quando comparado com as outras temperaturas de sinterização.

- A variação da temperatura sinterização de 1200 para $1300^{\circ} \mathrm{C}$ resultou em aumento considerável do tamanho de grão.

- Pode-se prever que a homogeneização obtida na sinterização poderá ser otimizada reduzindo-se o tamanho das partículas de $\mathrm{Nb}$.

\section{Agradecimentos}

Os autores agradecem à FAPERJ (processo E-26/111.678/2013) pelo apoio financeiro, a Rafael de Abreu Vinhosa e Simone Kessler de Barros pelo apoio técnico na preparação de amostras metalográficas, a João Carlos V. Silva pelo apoio técnico nas atividades de extrusão angular em canal e a Amanda Maria Paes Trindade, Agemar de Paula Filho e Fabio Henrique Silva pelo apoio técnico nos procedimentos experimentais de metalurgia do pó.

\section{REFERÊNCIAS}

1 Brooks CR. Heat Treatment, Structure and Properties of Nonferrous Alloys. $2^{\mathrm{a}}$ edição. Metals Park: American Society for Metals (ASM); 1982.

2 Donachie Jr MJ. Titanium, a Technical Guide, $3^{\text {a }}$ edição. Materials Park: ASM International; 2007.

3 Khan, MA, Williams RI, Williams DF. In-vitro corrosion and wear of titanium alloys in the biological environment. Biomaterials. 1996; 17 (22): 2117-2126.

4 Evans EJ. Cell damage in vitro following direct contact with fine particles of titanium, titanium alloy and cobalt-chrome-molybdenum alloy. Biomaterials. 1994; 15 (9): 713717.

5 Hon YH, Wang JY, Pan YN. Composition/phase structure and properties of TitaniumNiobium alloys. Materials Transactions. 2003; 44 (11): 2384-2390. 
Balcerzak AT, Sass SL. The formation of the $\omega$ phase in Ti-Nb Alloys. Metallurgical Transactions. 1972; 3 (6): 1601-1605.

7 Ninomi M. Mechanical properties of biomedical titanium alloys. Materials Science and Engineering A. 1998; 243: 231-236.

8 Anekem S, Greene CA. Recent developments in microstructure/property relationships of beta titanium alloys: Materials Science and Engineering A. 1999; 263: 127-131.

9 Ninomi M. Recent metallic materials for biomedical applications. Metallurgical and Materials Transactions A. 2002; 33-A (3): 477-488.

10 Buly RL, Huo MH, Salvati E, Brien W, Bansal M. Titanium wear debris in failed cemented total hip arthroplasty: An analysis of 71 cases. The Journal of Arthroplasty. 1992; 7 (3): 315-323.

11 Lombardi AV, Mallory TH. Aseptic loosening in total hip arthroplasty secondary to osteolysis induced by wear debris from titanium-alloy modular femoral heads, Journal of Bone and Joint Surgery. 1989; 71 (9): 1337-1342.

12 Grupo Setorial de Metalurgia do Pó, A metalurgia do Pó: alternativa econômica com menor impacto ambiental. São Paulo: Metallum Eventos Técnicos e Científicos; 2009.

$13 \mathrm{Wu} \mathrm{X}$, Xia K. Synthesis of bulk materials by equal channel angular consolidation of particles. Materials Science Forum. 2006; 503-504: 233-238.

$14 \mathrm{Ng} \mathrm{HP}$, Haase C, Lapovok R, Estrin Y. Improving sinterability of Ti-6AI-4V from blended elemental powders through equal channel angular pressing. Materials Science and Engineering A: 2013; 565: 396-404.

15 Carvalho SS, Lunz JL, Guzela LR, Licona KPM, Barros TS, Ribeiro AA, Pereira LC, Garcia de Blas JC, Oliveira MV, Barbosa C. Processamento e Caracterização de Titânio Submetido a Metalurgia do Pó e Extrusão Angular em Canal. In: $69^{\circ}$ Congresso Anual da ABM; 2014 jul 21-25; São Paulo, Brasil. São Paulo: Associação Brasileira de Metalurgia, de Materiais e Mineração; 2014: Artigo 24330.

16 Ribeiro AA, Balestra RM, Barros TS, Carvalho SS, Guzela LR, Barbosa C, Abud IC, Oliveira MV, Garcia de Blas JC, Pereira LC. Characterization of Titanium Powders Processed at Different Temperatures. Materials Science Forum. 2014; 802: 512-517.

17 Iwahashi Y, Wang J, Horita Z, Nemoto M, Langdon TG. Principle of equal-channel angular pressing for the processing of ultra-fine grained materials. Scripta Materialia. 1996; 35:143-146.

18 Furukawa M, Iwahashi Y, Horita Z, Nemoto M, Langdon TG. The shearing characteristics associated with equal-channel angular pressing. Materials Science and Engineering A. 1998; 257: 328-332.

19 WK19864 Revision of B311 - 93(2002)e1 Test Method for Density Determination for Powder Metallurgy (P/MP/M Materials Containing Less Than Two Percent Porosity.

20 Griza S, Sá DHGS, Batista, WW, Garcia de Blas JC, Pereira LC. Microstructure and mechanical properties of hot rolled TiNbSn alloys. 2014; 56: 200-208.

21 Oliveira CSS, Griza S, Oliveira MV, Ribeiro AA, Leite MB. Study of the porous Ti35Nb alloy processing parameters for implant applications. Powder Technology. 2015; 281:91-98. 